\title{
The Spectrum of Data sharing Policies in Neuroimaging Data Repositories
}

\author{
Anita S. Jwa ${ }^{1} \&$ Russell A. Poldrack ${ }^{1}$ \\ ${ }^{1}$ Department of Psychology, Stanford University \\ 450 Serra mall, Building 420 \\ Stanford, CA 94305
}




\begin{abstract}
Sharing data is a scientific imperative that accelerates scientific discoveries, reinforces open science inquiry, and allows for efficient use of public investment and research resources. Considering these benefits, data sharing has been widely promoted in diverse fields and neuroscience has been no exception to this movement. For all its promise, however, the sharing of human neuroimaging data raises critical ethical and legal issues, such as data privacy. Recently, the heightened risks to data privacy posed by the exponential development in artificial intelligence and machine learning techniques has made data sharing more challenging; the regulatory landscape around data sharing has also been evolving rapidly. Here we present an in-depth ethical and regulatory analysis that will examine how neuroimaging data are currently shared against the backdrop of the relevant regulations and policies and how advanced software tools and algorithms might undermine subjects' privacy in neuroimaging data sharing. This analysis will inform researchers on responsible practice of neuroimaging data sharing and shed light on a regulatory framework to provide adequate protection of neuroimaging data while maximizing the benefits of data sharing.
\end{abstract}




\section{Introduction}

Sharing data is an essential aspect of the scientific method (Alter \& Gonzalez, 2018; Ross, Iguchi, \& Panicker, 2018). It accelerates scientific advancement by creating large data sets through combining multiple data sources and by enabling the investigation of novel hypotheses not conceivable at the time of the initial data collection. Data sharing also makes possible the verification and replication of scientific findings, which reinforces open scientific inquiry and increases public trust in scientific research. It further allows more efficient use of public investment and research resources by preventing redundant data collection. Moreover, data sharing is not just a scientific imperative but also is an ethical duty for researchers to honor the efforts of human research subjects and to maximize the potential benefits of these efforts (Brakewood \& Poldrack, 2013).

Considering these benefits, data sharing has been widely endorsed in diverse fields, and neuroscience has been no exception to this movement (Breeze, Poline, \& Kennedy, 2012; Choudhury, Fishman, McGowan, \& Juengst, 2014; Mennes, Biswal, Castellanos, \& Milham, 2013; Poldrack \& Gorgolewski, 2017; Poline et al., 2012). The amount of shared neuroimaging data has been greatly increased during the last few decades and a number of neuroimaging data sharing initiatives and platforms have been established to promote research on shared data. However, at the same time, data sharing practice in neuroimaging has encountered several challenges, and significant efforts have been made to overcome these challenges.

Initially, technical and infrastructural difficulties presented significant challenges. Given the size of neuroimaging data, building an infrastructure to store and share a massive amount of data poses a substantial barrier in data sharing. It is also crucial to build a field-wide consensus on how to organize and share neuroimaging data. With the aid of recent technological advancements in data storage, such as commercial cloud computing platforms, along with the exponential increase in computing power, it has become technically straightforward to share large neuroimaging data sets. Efforts to develop a set of community standards on format and structure of neuroimaging datasets (e.g., Brain Imaging Data Structure (Gorgolewski et al., 2016a)) have also substantially facilitated neuroimaging data sharing. Systems to assign unique identifiers to individual subjects or datasets have also made shared data more easily findable.

Another major challenge lies in motivating researchers to share their data. At the beginning, researchers were worried that if data were shared, other researchers might analyze the data and publish the results before them or might reveal errors in their initial data analysis (Nichols et al., 2017). Along with the extra costs to prepare and manage shared data, uncertainty around how to credit data sharing in terms of citations and academic promotions has also dissuaded researchers from sharing. The unfortunate failure of the fMRI Data Center (fMRIDC), an ambitious pioneering neuroimaging data sharing project in early 2000, was in part due to the strong opposition from the discomforted researchers (Van Horn \& Gazzaniga, 2013). Since then, to address these concerns, data repositories have imposed a grace period before they make data publicly available, which gives data contributors time to publish their research and protects them from being "scooped" by others. In addition, funding agencies have begun to provide financial support for management and sharing of data as part of a research grant. Mechanisms to acknowledge efforts to collect and share data, such as data papers and data citation, have also been introduced in order to encourage the practice of sharing data.

Sharing neuroimaging data also raises critical ethical concerns (Brakewood \& Poldrack, 2013). If derived from human subjects, the data should be shared in accordance with the ethical principle of 
protecting human subjects. Yet obtaining fully Informed consent for secondary data analyses is challenging because these analyses might not yet have been developed or even conceived of at the time of initial data collection. Rigorous privacy and security measures should also be in place when sharing data to minimize risks to subjects' privacy and confidentiality of data. Neuroimaging researchers have applied various deidentification methods, such as removal of direct identifiers (i.e., name and address) from metadata or defacing structural MR images, to protect subjects' privacy (see e.g., Bischoff-Grethe et al., 2007).

Unlike the infrastructural and motivational challenges, however, these ethical challenges around data sharing have become increasingly more difficult and complex to navigate, as the regulatory landscape around data sharing has been evolving rapidly, and recent advances in artificial intelligence and machine learning techniques pose heightened risks to data privacy (The Federal Policy for the Protection of Human Subjects, 2018; Peloquin, DiMaio, Bierer, \& Barnes, 2020; White, Blok, \& Calhoun, 2020). Federal regulations and funding agencies' policies on data sharing have provided some guidance on how to respect subjects' wishes regarding the future use of their data and how to protect data privacy, but they often cause more uncertainty than clarity in the actual practice of data sharing. The emergence of novel software tools and algorithms, such as facial reconstruction techniques (Schwarz et al., 2019; Ravindra \& Grama, 2019; Abramian and Eklund, 2019), is also particularly concerning due to their potential ability to invalidate conventional de-identification methods and to re-identify subjects in neuroimaging datasets.

As funding agencies have begun to implement their own policies that require investigators to share data collected as part of funded research (National Institutes of Health (NIH), 2020a; National Institutes of Mental Health (NIMH), 2019; The Brain Research Through Advancing Innovative Neurotechnologies Initiative (BRAIN), 2019), sharing data should become increasingly common in the field of neuroimaging, and the body of shared neuroimaging data will likely continue to grow substantially. Thus, there is a pressing need to unravel the ethical concerns to promote more responsible sharing of neuroimaging data. Here we present an in-depth ethical and regulatory analysis that will examine how neuroimaging data are currently shared against the backdrop of the relevant regulations and policies and how advanced software tools and algorithms might undermine subjects' privacy in neuroimaging data sharing. This analysis will first review ethical principles for the protection of human subjects in the context of data sharing and provide an overview of the regulatory landscape regarding data sharing in the United States. Then it will survey the current practice of data sharing in existing neuroimaging data repositories, focusing on restrictions on access to and subsequent use of data in these repositories. Finally, it will examine novel technological threats of re-identifying neuroimaging data and the implications these threats could have on data sharing practices and policies.

\section{Ethical Challenges in Neuroimaging Data Sharing}

Sharing human subject data for secondary analysis has been one of the primary topics that arises in discussions concerning the protection of human research subjects. The Ethical Principles and Guidelines for the Protection of Human Subjects of Research (also known as the Belmont Report) laid out three core ethical principles, namely, respect for persons, beneficence, and justice (National Commission for the Protection of Human Subjects, 1978).

The principle of respect for persons requires human subjects to be treated as autonomous agents and that human subjects be provided special protection should they possess diminished cognitive abilities. Abiding to this principle demands researchers to assure that prospective subjects are capable of making a decision as to whether to participate in a study and that subjects are given sufficient information about the 
study, such as purpose and procedure of the study, to make informed decisions. Informed consent should include whether data collected from the subjects will be shared for secondary analysis. However, movement to open access and open data sharing calls for a reassessment of the common interpretation of this principle (Ross, Iguchi, \& Panicker, 2018). In open data sharing, it is difficult to predict and control how neuroimaging data will be analyzed, by whom, and for what purposes once the data are shared through data repositories, and therefore it may not be possible to obtain informed consent, as in a traditional sense, for a specific secondary analysis on the subjects' data. Rather, data sharing requires broad consent that allows a range of possible analyses that are not pre-specified.

The principle of beneficence imposes an obligation for researchers to protect subjects by minimizing risks of harm and maximizing potential benefits to the subjects. It requires potential benefits to outweigh any risks of harm in the conducting of a research study. In weighing the risks against the benefits, researchers should consider both the probability and magnitude of harm (Alter \& Gonzalez, 2018), and this principle can also be extended to consider the benefits beyond an individual subject participating in a study (Ross, Iguchi, \& Panicker, 2018). Under this principle, data sharing becomes an ethical duty for researchers to promote societal good from existing data, such as benefits to public health and welfare, given that subjects are protected from the risks of harm. In the context of neuroimaging data sharing, the primary risks to subjects include the violation of privacy and breach of confidentiality. Privacy and confidentiality differ in a sense that the former applies to persons, whereas the latter applies to personal information and data. Privacy refers to human subjects' interests in controlling "when, how, and to what extent information about them is communicated to others" (Westin, 1968). An extension of privacy, confidentiality pertains to information about subjects disclosed to researchers with the expectation that the information would not be revealed to others (Cooper \& McNair, 2015). The duty to maintain confidentiality arises out of researchers' agreement with subjects about how the subjects' information will be handled, managed, and disseminated to prevent unauthorized disclosure of the information. When sharing human subject data, the principle of beneficence requires researchers to de-identify data to minimize the risk to privacy and employ rigorous data security measures to prevent breach of confidentiality.

The principle of justice requires fair distribution of the benefits and burdens of human subject research. It primarily relates to equitable selection of subjects for a research study to ensure that subjects do not bear risks to benefit other people. Sharing data could actually promote justice by pooling data from a diverse population, and it facilitates the distribution of the benefits of research findings by increasing the generalizability of these findings.

\section{Overview of Regulatory Landscape in the United States}

As a means of implementing the ethical principles of human subject protection in the Belmont Report, a multitude of federal regulations and policies have been stipulated that serve to protect human subjects participating in research studies. This section will review these regulations and policies. It will specifically focus on their application to the sharing of and the secondary analysis of neuroimaging data within the research context in an effort to provide a general overview of the regulatory framework in the United States.

\section{a. Common Rule}

The Department of Health and Human Services (HHS) codified the basic regulations to protect human subjects in research. These regulations, better known as "Common Rule," have been adopted by many other federal departments and operate as the standard for ethical conduct of government-funded research (The 
Federal Policy for the Protection of Human Subjects, 2018). The Common Rule was recently revised in 2018 to provide additional regulatory options for conducting research involving human subjects. ${ }^{1}$ For human subject research, the Common Rule requires researchers to obtain informed consent from subjects and the Internal Review Board (IRB) to review and approve research activity.

Analysis of shared data is considered secondary research, which refers to research use of information or biospecimens originally collected for research studies other than the proposed one or collected for non-research purposes. Under the Common Rule, secondary research that obtains, uses, studies, or analyzes identifiable private information falls under the category of human subject research and is thus subjected to the Common Rule requirements. Identifiable private information is that for which the identity of the subject is, or may readily be, ascertained by the investigator or is associated with the information. $^{2}$ Thus, if shared neuroimaging data contain identifiable information, then investigators conducting research on these data must obtain informed consent from individuals to whom the information pertains and undergo IRB review for the research activity.

Under certain circumstances, secondary research with identifiable private information can be exempted from the Common Rule requirements. This includes when the information is publicly available or when the information is recorded by the investigator in such a manner that the identity of the human subjects cannot be readily ascertained. ${ }^{3}$ The 2018 revision of the rule introduced broad consent as a new option for investigators to conduct secondary research. Broad consent is intended to allow subjects to exercise their autonomy in controlling the use of information pertaining to them while facilitating future research use of the information when the details of such future research have not yet been specified. ${ }^{4}$ If broad consent is obtained at the time of initial collection of identifiable information, storage, management, and use of this information for secondary research are exempted under the Common Rule, and only limited IRB review is required. ${ }^{5}$ In cases in which these exemptions are not applicable, investigators can still seek waiver of informed consent to conduct the research. ${ }^{6}$

Unlike secondary use of identifiable private information, research on nonidentifiable private information does not constitute human subject research under the Common Rule. In other words, if secondary research involves only coded private information and investigators cannot readily ascertain the identity of the individual(s) to whom the information pertains, the Common Rule requirements, informed consent and IRB review, do not apply (Office of Human Research Protections (OHRP), 2008). In most neuroimaging data shared through existing repositories, identifiable information is deleted or replaced with

\footnotetext{
1 Common Rule defines human subject as "a living individual about whom an investigator conducting research that obtains information or biospecimens through intervention or interaction with the individual and uses, studies, or analyzes the information or biospecimens; or obtains, uses, studies, analyzes, or generates identifiable private information or identifiable biospecimen." 45 CFR 46.102(e)(1).

245 CFR 46.102(e)(5).

345 CFR 46.104(d)(4); There are two other circumstances in which consent is not required for secondary research under this exemption - if the use of identifiable information is regulated under Health Insurance Portability and Accountability Act or if research is conducted by, or on behalf of, a federal agency using data collected or generated by the government for non-research purposes and the data is protected by federal privacy standards.

${ }^{4}$ In addition to some elements from standard informed consent (e.g., reasonably foreseeable risks, potential benefits to subjects or others, confidentiality safeguards, voluntary participation), broad consent includes unique requirements regarding secondary use of data, such as a general description of the types of research that many be done, a description of the identifiable materials that might be used, whether there might be sharing, and with what types of institutions or researchers (45 CFR 46.116(d)).

545 CFR 46.104(d)(7), (8); 45 CFR 46.111(a)(8).

${ }^{6} 45$ CFR 46.116(f)(3).
} 
a code (e.g., a number, letter, or symbol), and thus, secondary research on these nonidentifiable neuroimaging data is not considered research involving human subjects.

The Common Rule has its own enforcement mechanism for researchers and institutions that failed to meet the requirement for human subject protections, including suspension of research or termination of federal funding. ${ }^{7}$ However, the rule does not provide private cause of action for violation of the regulation, and currently, subjects who experience harm as a result of their participation in a research study are left without any legal remedies (Koch, 2015). ${ }^{8}$

\section{b. Health Insurance Portability and Accountability Act}

The Health Insurance Portability and Accountability Act (HIPAA) was enacted to improve the efficiency and effectiveness of the healthcare system and includes provisions - the Privacy Rule and Security Rule to establish national standards to protect medical records and other personal health information. The HIPAA Privacy Rule defines and limits the circumstances in which an individual's protected health information may be used or disclosed by certain covered entities, such as health plans, health care providers, or healthcare clearinghouses (U.S. Department of Health and Human Services (HHS), 2003a). Research institutions, such as a college or university, could also qualify as covered entities, if they have components that perform covered functions (e.g., providing health care at their medical facilities) (U.S. Department of Health and Human Services (HHS), 2008). ${ }^{9}$ As discussed above, most federally funded research involving human subjects is regulated under the Common Rule, which provides protections for the privacy of subjects and for the confidentiality of information collected for a research. The Privacy Rule builds upon these protections, and if a researcher conducting a neuroimaging study is a part of a covered entity, she would also be subjected to the Privacy Rule.

Under the HIPAA, protected health information (PHI) is defined as individually identifiable health information that a covered entity holds or transmits. ${ }^{10}$ Here, individually identifiable health information refers to information related to the individual's past, present, or future physical or mental health/conditions and identifies the individual or for which there is a reasonable basis to believe that it could be used to identify the individual. ${ }^{11}$ Individually identifiable health information includes many common identifiers (e.g., name, address, birth date, Social Security number). A covered entity may not use or disclose PHI except in instances in which the Privacy Rule requires or permits it to do so. For example, use and disclosure of PHI for research purposes is permitted with documentation of an alteration (or a waiver) of individual's authorization approved by IRB. ${ }^{12}$ The Privacy Rule also permits the research use of PHI, if certain, specified

\footnotetext{
745 CFR $\$ 46.123$.

${ }^{8}$ The U.S. did not sign on to the 7 th edition of the Declaration of Helsinki that has a new general principle regarding the compensation for research-related injuries; "[a]ppropriate compensation and treatment for subjects who are harmed as a result of participating in research must be ensured (World Medical Association, 2008)." The Presidential Commission for the Study of Bioethical Issues has made recommendations regarding the need for compensation for research-related injuries, but there has been no clear response from the federal government (Presidential Commission for the Study of Bioethical Issues, 2011).

${ }^{9}$ A higher-education institution that performs both covered and non-covered functions may choose to be a hybrid entity under the HIPAA (45 CFR §164.103). To become a hybrid entity, an institution must define and designate its health care component, separate from other components that perform non-covered functions, and the HIPAA Privacy or Security Rule requirements would apply only to the health care component.

${ }^{10} 45$ CFR $\S 164.103$

${ }^{11} \mathrm{Id}$.

1245 CFR $§ 164.512(i)$
} 
unique identifiers of individuals have been removed ("limited data set") and the recipient enters into a data use agreement that promises to safeguard the PHI within the data set. ${ }^{13}$

However, the Privacy Rule also allows a covered entity to use PHI to create information that is not individually identifiable, ${ }^{14}$ and the use or disclosure of this de-identified information is no longer regulated under the HIPAA. ${ }^{15}$ Unlike the Common Rule that provides only a general definition of nonidentifiable private information, HIPAA sets out two specific standards to determine de-identification of PHI. One is a formal determination by a qualified statistician that the risk is extremely low that the information could be used - alone or in combination with other reasonably available information - to identify an individual who is a subject of the information. ${ }^{16}$ The other, known as the safe harbor method, entails the removal of 18 unique identifiers from PHI provided that the covered entity has no actual knowledge that the remaining information could be used to identify the individual. ${ }^{17}$ Therefore, de-identified neuroimaging data that do not contain these identifiers can be shared and analyzed without restrictions under the HIPAA regulations. This safe harbor method has been widely accepted and used by researchers, even if they are not subjected to HIPAA, as a conventional way to de-identify data.

The HIPAA Security Rule has stipulated security standards for the protection of PHI that is held or transferred in electronic form (e-PHI) by covered entities. These standards are intended to operationalize the protections provided in the Privacy Rule (U.S. Department of Health and Human Services (HHS), 2003b). The Security Rule requires covered entities to implement reasonable and appropriate administrative, technical, and physical safeguards to ensure the confidentiality, integrity, and availability of e-PHI. ${ }^{18}$ For example, covered entities must perform a risk analysis to evaluate potential security risks to e-PHI and to put appropriate security measures in place to prevent unauthorized disclosure of e-PHI. ${ }^{19}$ Again, the Security Rule does not apply when covered entities create, receive, maintain, or transmit deidentified PHI.

HIPAA imposes substantial financial penalties to covered entities that failed to comply with its regulations, but similar to the Common Rule, HIPAA does not provide a private cause of action that allows for an individual to sue a covered entity for violation of the HIPAA (U.S. Department of Health and Human Services (HHS), 2013). ${ }^{20}$ Nevertheless, an individual can file a health information privacy complaint to the

\footnotetext{
${ }^{13} 45$ CFR §164.514(e)(2),(4); These identifiers include (i) names; (ii) postal address information, other than town or city, State, and zip code; (iii) telephone numbers; (iv) fax numbers; (v) electronic mail addresses; (vi) social security numbers; (vii) medical record numbers; (viii) health plan beneficiary numbers; (ix) account numbers; (x) certificate/license numbers; (xi) vehicle identifiers and serial numbers, including license plate numbers; (xii) device identifiers and serial numbers; (xiii) web Universal Resource Locators (URLs); (xiv) internet Protocol (IP) address numbers; (xv) biometric identifiers, including finger and voice prints; and (xvi) full face photographic images and any comparable images.

1445 CFR $\$ 164.502(d)$

15 "Health information that does not identify an individual and with respect to which there is no reasonable basis to believe that the information can be used to identify an individual is not individually identifiable health information." 45 CFR $\$ 164.514(\mathrm{a})$

1645 CFR $\$ 164.514(b)(1)$

${ }^{17} 45$ CFR $\S 164.514(\mathrm{~b})(2)$

${ }^{18}$ Under the Security Rule, covered entities should ensure that e-PHI is not available or disclosed to unauthorized persons (confidentiality), e-PHI is not altered or destroyed in an unauthorized manner (integrity), and e-PHI is accessible and usable on demand by an authorized person (availability). 45 CFR $§ 164.304,306$.

${ }^{19} \S 164.308(\mathrm{a})(1)(\mathrm{ii})(\mathrm{A})$.

${ }^{20}$ Under the Health Information Technology for Clinical and Economic Health (HITECH) Act, part of the American Recovery and Reinvestment Act of 2009, State Attorneys General has the authority to bring civil actions on behalf of state residents for violations of the HIPAA Privacy and Security Rules. State Attorneys General can obtain
} 
Office of Civil Rights (OCR). ${ }^{21}$ The OCR will conduct an investigation upon receiving the complaint and issue a letter regarding the resolution of the complaint. If a covered entity was found to have violated HIPAA, it must voluntarily comply with the HIPAA rules, take corrective action, or agree to a settlement (U.S. Department of Health and Human Services (HHS), 2017a). A covered entity that fails to take satisfactory action to resolve the matter may be subject to civil monetary penalties or criminal prosecution (U.S. Department of Health and Human Services (HHS), 2017b).

\section{c. The NIH Data Sharing Policy}

The National Institutes of Health (NIH), one of the major federal funding agencies for biomedical research in the United States, has published its own policy to promote the sharing of data, including neuroimaging data, generated from research funded or conducted by the NIH. This policy, which will be effective from January 2023, requires researchers applying for NIH funding to submit a Data Management and Sharing Plan (DMS plan) for any NIH-funded or conducted research that will generate scientific data (National Institutes of Health (NIH), 2020a). ${ }^{2223}$ While acknowledging the importance and benefits of data sharing, the NIH has emphasized the protection of rights and privacy of human subjects who have participated in NIH-funded research.

The policy does not introduce new additional requirements regarding for the protection of human subject data beyond existing laws and regulations (e.g., the Common Rule or Certificates of Confidentiality ${ }^{24}$ ), but researchers must outline in their DMS plan how privacy, rights, and confidentiality of human subjects will be protected. The policy provides three concepts to consider in sharing human subject data: 1) how to address data management and sharing in the informed consent process, 2) whether there are any limitations on subsequent use of data should be communicated to those individuals or entities preserving and sharing the data, and 3) even when data have been de-identified and lack explicit limitations on subsequent use, whether access to these data should still be controlled (National Institutes of Health (NIH), 2020a). Yet, it is important to note that this policy does not preclude the open sharing of data from human subjects in ways that are consistent with consent practices, established norms, and applicable laws.

The policy also provides a guidance for researchers on how to choose a proper data repository by delineating the desirable characteristics of a repository, including that having security measures in place to prevent unauthorized access to, modification of, or release of sensitive data and having the capabilities to ensure confidentiality of data (National Institutes of Health (NIH), 2020b). In specific regard to repositories

damages on behalf of state residents or to enjoin further violations of the HIPAA Privacy and Security Rules (U.S. Department of Health and Human Services (HHS), 2017b).

2145 CFR $\$ 160.306$.

22 Previous data sharing policy issued in 2003 applied to research studies with direct costs of more than $\$ 500,000$, but the new policy released in 2020 substantially expands the scope of data sharing to all research funded by the NIH regardless of the amount of funding provided (National Institute of Health (NIH), 2003).

${ }^{23}$ Elements that need to be addressed in the DMS plan includes (1) types and estimated amount of data, (2) related tools, software and/or code needed to access or manipulate shared data, (3) standards to be applied to data and meta data, (4) plans and timelines for data preservation and access, (5) access, distribution, or reuse consideration (e.g., informed consent or privacy and confidentiality protection consistent with applicable laws and regulations), and (6) oversight of data management and sharing (National Institute of Health (NIH), 2020a).

${ }^{24}$ Certificate of Confidentiality $(\mathrm{CoC})$ is issued to persons engaged in biomedical, behavioral, clinical, or other research - funded wholly or in part by $\mathrm{NIH}$, in which identifiable, sensitive information is collected. CoCs protect the privacy of research subjects by prohibiting disclosure of identifiable, sensitive research information to anyone not connected to the research except when subject consents or in a few other specific situations. https://www.hhs.gov/ohrp/regulations-and-policy/guidance/certificates-of-confidentiality/index.html 
storing human subject data, the policy lists additional considerations, for example, whether a repository restricts access to and use of data consistent with subjects' consent; communicates and enforces data use restrictions, such as preventing re-identification or redistribution to unauthorized users; and implements appropriate approaches (e.g., tiered access, credentialing of data users, security safeguards against potential breaches) to protect human subject data from inappropriate access (National Institutes of Health (NIH), 2020b).

\section{d. The NIMH Data Sharing Policy}

In 2019, the National Institute of Mental Health (NIMH), an NIH institute for research on mental disorders, published a Notice of Data Sharing Policy (National Institutes of Mental Health (NIMH), 2019). It requires researchers who are funded by NIMH to deposit their data, including neuroimaging data, to the NIMH Data Archive (NDA). All applications involving human subjects that are submitted to or referred to NIMH after January 1, 2020 are expected to include a Resource Sharing Plan as part of the application. ${ }^{25}$ A single NDA collection containing the data associated with each grant award will be established, and data from the award are expected to be submitted every six months. Submitted data will generally be held in a private enclave until papers using the data have been accepted for publication or until the end of the award period (including the first no-cost extension), whichever occurs first. In addition to submitting the data associated with a grant award, researchers are expected to separately submit to the NDA the specific data that were used for each resulting publication by creating an NDA Study to promote rigor and reproducibility of research. The NIMH also strongly encourages researchers to deposit their data from the NIMH-funded research to other appropriate repositories, such as the Brain Research Through Advancing Innovative Neurotechnologies (BRAIN) Initiative data archives.

\section{e. The BRAIN Initiative Data Sharing Policy}

Particularly relevant to our analysis, the BRAIN Initiative, a large-scale trans-agency effort to support research on human brain and function, also released a notice of its data sharing policy in 2019 (The Brain Research Through Advancing Innovative Neurotechnologies Initiative (BRAIN), 2019). For all BRAIN Initiative applications submitted after March 1, 2020, applicants are required to include a Resource Sharing Plan as part of the application and to submit their data to one of the designated archives established to hold data collected as part of BRAIN Initiative-funded research, including OpenNeuro, a repository for neuroimaging data. ${ }^{26}$ Similar to the NIMH data sharing policy, applicants are expected to submit data to the archives every six months, which will be held in a private enclave until papers using the data have been published or until the end of the award period. For research that involves human subjects, the Resource Sharing Plan should have a description of whether and how the consent obtained from the subjects to collect

\footnotetext{
25 The elements of Resource Sharing Plan include 1) a summary of the data that will be shared 2) a description of the standard(s) and/or data dictionaries that will be used to describe the data set, and 3) the proposed schedule to validate that the data are compliant with the data dictionary that is being used (National Institute of Mental Health (NIMH), 2019).

${ }^{26}$ A Resource Sharing Plan should include a summary of the data that will be shared, a description of the standard(s) that will be used to describe the data set, the data archive(s) that will house the data, the proposed timeline for submitting data to the archive and sharing data with the research community. Costs associated with preparing and submitting data to a data archive should also be included in grant applications (The Brain Research Through Advancing Innovative Neurotechnologies Initiative (BRAIN), 2019).
} 
data will limit future secondary research that can be done with the data. The notice further encourages applicants to use consent that allows for broad data sharing whenever possible.

The review of regulatory protections for human subjects indicates that research on shared neuroimaging data that contain identifiable private information be subject to the Common Rule requirements. If a researcher or a research unit is part of a covered entity under the HIPAA, sharing neuroimaging data with PHI should also comply with the HIPAA Privacy and Security Rule. However, neuroimaging data that have had identifiable information removed or coded in a way that makes the data nonidentifiable under the Common Rule or that are de-identified under the HIPAA can be shared without restrictions under these regulations. Funding agencies' data sharing policy also requires researchers to consider how to protect human subjects' privacy and the confidentiality of data derived from human subjects and to ensure that researchers conducting secondary analyses should abide by any additional limitations on subsequent use of data.

\section{Data Sharing Practice in Existing Neuroimaging Data Repositories}

Laws and regulations have stipulated general compliance standards for adequate data protection but examining how neuroimaging data are currently shared in data repositories can help us to understand the actual practice of data sharing in the field of neuroimaging. This section will survey data sharing practices in existing neuroimaging data repositories, focusing on restrictions on access to and subsequent use of data shared through these repositories.

\section{a. What types of neuroimaging data are shared?}

Existing neuroimaging data sharing initiatives or platforms offer different types of neuroimaging data, which involve varying levels of privacy risks. Some repositories only share peak coordinates of brain regions that showed statistically significant effects in published neuroimaging studies (e.g., The BrainMap project (Vanasse et al., 2018) and the Neurosynth project (neurosynth.org)). These coordinate-based data can be used to reconstruct the activation map in the studies. The next level is sharing unthresholded maps or network maps (e.g., Neurovault (Gorgolewski et al., 2016b)), which allows researchers to both examine results of the original study and conduct meta-analysis on subthreshold effects in brain regions beyond the peak coordinates reported in publications. Research on coordinate-based data or statistical maps does not pose significant privacy risks because they only provide group-level information. However, sharing raw individual-level data, such as NIfTI or DICOM files, could provide greater potential by enabling researchers not only to reanalyze the initial results but also to investigate new hypotheses beyond the ones tested in the original study. OpenNeuro (openneuro.org), International Neuroimaging Data-Sharing Initiative (INDI) (fcon_1000.projects.nitrc.org), Human Connectome Project (HCP) (Van Essen et al., 2013), and Alzheimer's Disease Neuroimaging Initiative (ADNI) (Jack et al., 2008) are some of the repositories that store and share raw (or preprocessed) individual structural, resting-state, or functional MRI data.

\section{b. How are neuroimaging data shared?}

The survey of existing neuroimaging data repositories shows a wide spectrum of data sharing practices. Some repositories offer fully open sharing without any limitations attached, whereas others impose certain restrictions on access to and subsequent use of data under their data sharing policy or data use agreement 
(Table 1). The level of restrictions in these repositories varies depending on the sensitivity of data and other relevant factors, such as whether original contributors still retain some control over shared data (e.g., by employing strict acknowledgment requirement) or whether a federal agency (e.g., NIH or FDA) is involved as a governing body of a repository.

$<$ Table 1: Current practice of data sharing in existing neuroimaging data repositories>*

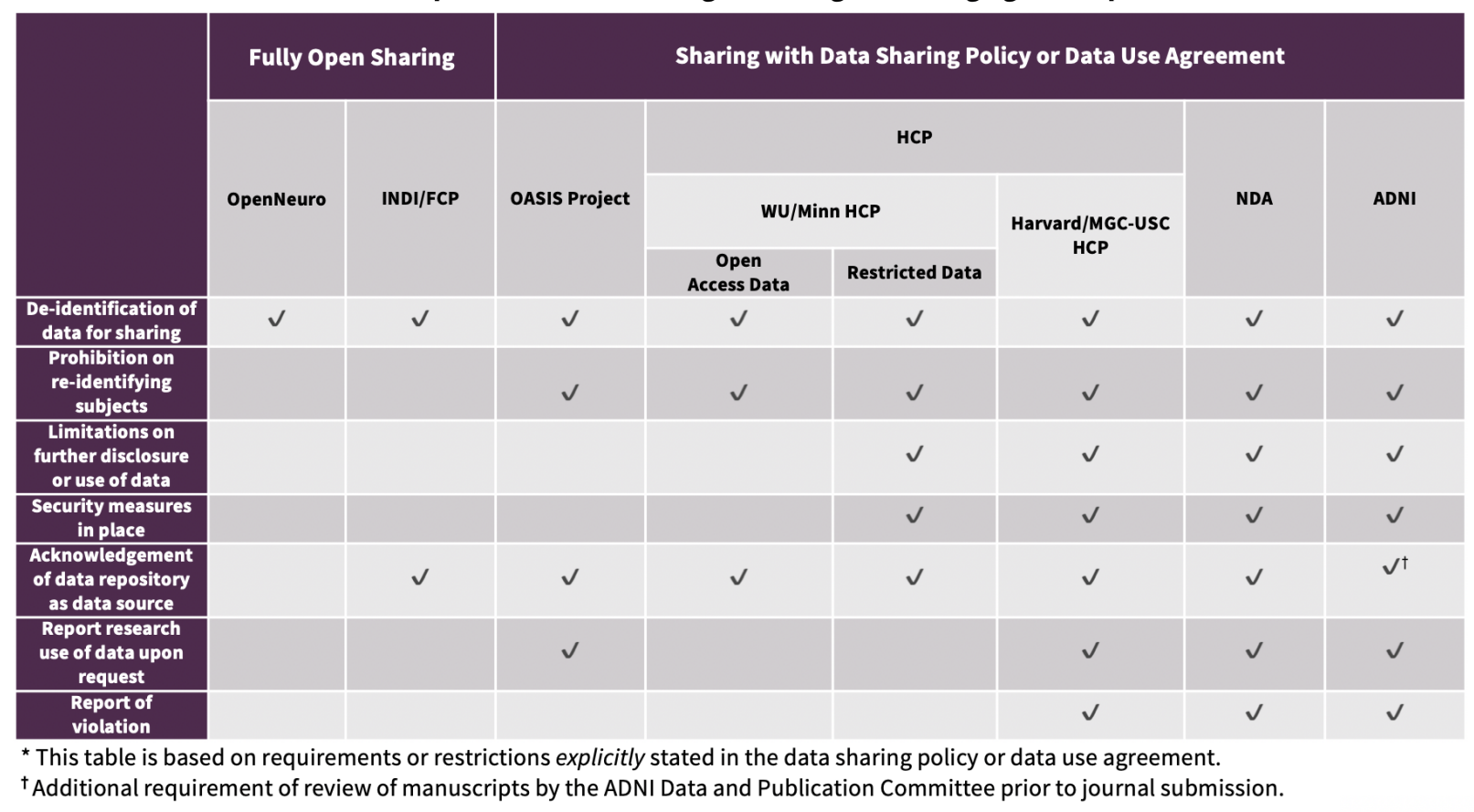

\section{i. Repositories offer fully open sharing}

Most repositories that support fully open sharing host neuroimaging data under certain Creative Commons (CC) licenses. For example, OpenNeuro (openneuro.org) requires researchers to agree that raw individuallevel data will be shared under Creative Commons CC0 license by waiving copyright and all other related rights to the data with a grace period of 36 months from the upload of the data. The CC0 license essentially puts the data in public domain, ${ }^{27}$ and thus, there is no restriction on the access and further use of data shared through OpenNeuro after the grace period. To protect subjects' privacy, researchers contributing data to OpenNeuro are also required to de-identify the data by deleting the unique identifiers as defined under the HIPAA, including facial features on structural images, and have ethical permission from their own institution to publicly share data. Other open sharing initiatives or platforms, such as 1,000 Functional Connectomes Project (FCP) (Biswal et al., 2010) and International Neuroimaging Data-Sharing Initiative (INDI) (fcon_1000.projects.nitrc.org), both prospectively and retrospectively, share data under Creative Commons, Attribution-NonCommercial-ShareAlike License. It is free to copy, redistribute, and adapt data, but unlike the $\mathrm{CC} 0$ license, data can be shared only for non-commercial research purposes, and users must

\footnotetext{
${ }^{27}$ Creative Commons, CC0. https://creativecommons.org/share-your-work/public-domain/cc0/
} 
give appropriate credit for the shared data. ${ }^{2829}$ The INDI also has detailed guidelines for researchers to follow when preparing their data for sharing. ${ }^{30}$ Specifically, it requires that datasets do not contain any identifiable information in the images and image-headers to ensure HIPAA-compliant de-identification. Facial features should also be removed from any high-resolution images and each subject's ID must be changed to an anonymized numeric value provided by the INDI.

ii. Repositories have a data sharing policy or data use agreement (DUA)

Some neuroimaging data repositories require researchers who want to share their data or use shared data for secondary analysis to explicitly agree with their data sharing policy or data use agreements, which include various restrictions on data access and future use of data. This section will review these data sharing policies and data use agreements in select neuroimaging repositories.

\section{The Open Access Series of Imaging Studies (OASIS)}

The OASIS project (oasis-brains.org) archives neuroimaging datasets on cognitively normal and demented subjects (with mild to moderate Alzheimer's disease) that are freely available to the scientific community with relatively minimal requirements under its data use agreement. The DUA of the OASIS project sets out terms including that users should not attempt to establish the identity of human subjects, and should acknowledge the use of OASIS data when publicly presenting any results or algorithms that benefited from the data. ${ }^{31}$ Users could also be requested to provide updates on research use of data. Failure to abide by these terms will result in termination of the right to access and use OASIS data.

\section{The Human Connectome Project (HCP)}

The NIH Human Connectome Project is an effort to acquire and share data on the structural and functional connectivity of the brain to map the neural pathways that underlie human brain function. It established two research consortia to achieve this goal, the University of Washington St. Louis, University of Minnesota, and Oxford University Project (Van Essen et al., 2013) and the Harvard/Massachusetts General Hospital University of Southern California Project (humanconnectomeproject.org). These two consortia have different DUA for the use and sharing of data collected.

The WU/Minn HCP consortium developed an initial study, now known as the Young Adult HCP project, which shared data from about 1200 individuals. Subsequent extensions include the Lifespan HCP

\footnotetext{
${ }^{28}$ Creative Commons, Attribution-NonCommercial-ShareAlike 4.0 International https://creativecommons.org/licenses/by-nc-sa/4.0/; The standard INDI data sharing policy is Creative Commons, Attribution-NonCommercial-ShareAlike license, but some datasets in the INDI are shared under more or less restrictive licenses (e.g., Creative Commons, Attribution-Non-Commercial license in the ADHD-200 or Open Data Commons Public Domain Dedication and License(PDDL) in the Max Planck Institute Leipzig Mind-Brain-Body dataset) or under their own Data Use Agreement (e.g., Anatomical Tracings of Lesions After Stroke (ATLAS)).

${ }^{29}$ Other repositories have developed their own copyright policy - for instance, BrainMap, a repository that stores and shares coordinate-based data, makes its software and data available only for non-commercial purposes while allowing its coding scheme and taxonomy of experimental design available for use without restriction. It also requires that if BrainMap's data, software, or coding strategies are used in scientific publications, one or more relevant publications of the development team should be cited.

${ }^{30}$ International Neuroimaging Data-Sharing Initiative Data Contribution Guide. http://fcon_1000.projects.nitrc.org/indi/indi_data_contribution_guide.pdf

${ }^{31}$ The Open Access Series of Imaging Studies. Data Use Agreement. https://www.oasis-brains.org
} 
project and a number of disease-specific Connectome projects ${ }^{32}$ The Young Adult HCP data are released through the ConnectomeDB(db.humanconnectome.orge). Because this subproject includes subjects who are twins and siblings from extended families located in a limited geographical area, the potential for reidentification is increased. For this reason, the project implemented a two-tiered approach that distinguishes data that can be openly shared and those for which access should be restricted. Open access data essentially include all image data and most of the behavioral data stored in the database, and restricted data include information such as family structure (e.g., twin status) and age that could be used to re-identify subjects if combined with open access data. Along with the requirement of preventing users from reidentifying and contacting human subjects, the Data Use Terms for open access data emphasizes that the code linking data to PHI or any additional information about individual subjects will not be provided under any circumstances. ${ }^{33}$ It further specifies that users should comply with all relevant rules and regulations regarding the protection of human subjects (e.g., proposed research should be approved or exempted by IRB or Ethics Committee). Open access data, and any derived data, may be redistributed under the same terms, and users should acknowledge the WU/Minn HCP when publicly presenting results gained from the data. Again, failure to comply with the terms will cause termination of access to data. In order to obtain access to restricted data, users are required to abide by additional terms to protect subjects' privacy, such as prohibition of further distribution or sharing of data, secured access to data, and restrictions on the publication of data. ${ }^{34}$

The Harvard/MGH-USC HCP consortium also asks users to abide by its DUA in order to obtain access to shared data. ${ }^{35}$ Its DUA prohibits users from re-identifying subjects and disclosing data beyond the uses outlined in the DUA (e.g., purposes of scientific investigation, teaching, or the planning of clinical research studies) or delineated in the application by the users. It also requires users to employ appropriate administrative, physical, and technical security measures to prevent use or disclosure of the data other than as provided in the DUA and the application. Users should report any event of unauthorized use or disclosure, and they are expected to acknowledge and cite the HCP as the source of data in the publications.

\section{The National Institute of Mental Health (NIMH) Data Archive (NDA)}

The National Institute of Mental Health Data Archive (nda.nih.gov) was established to store and share data related to autism research but has evolved into an informatics platform that houses data collected as part of NIMH funded research. The archive also stores data from mental health research initiatives, such as the Adolescent Brain Cognitive Development (ABCD) Study (abcdstudy.org) and the Connectome Coordination Facility (CCF) (humanconnectome.org/lifespan-studies), and operates as an overarching database to provide a single process for gaining access to the data in these repositories. The NDA Data

\footnotetext{
${ }^{32}$ The data collected as part of Lifespan HCP project are housed in the National Institute of Mental Health Data Archive (NDA) and access to these data is governed by the Adolescent Brain Cognitive Development (ABCD). (NIMH Data Archive. Accessing Data from the May 2019 HCP Lifespan Data Release.

https://s3.amazonaws.com/NDARExternalResources/Documents/HCP+Data+Access+Instructions +1.0+Lifespan+R elease.pdf)

${ }^{33}$ WU-Minn HCP Consortium. Open Access Data Use Terms. https://www.humanconnectome.org/storage/app/media/data_use_terms/DataUseTerms-HCP-Open-Access26Apr2013.pdf

${ }^{34}$ WU-Minn HCP Consortium. Restricted Data Use Terms. https://www.humanconnectome.org/study/hcp-youngadult/document/wu-minn-hcp-consortium-restricted-data-use-terms

35 The Harvard/MGH-USC HCP consortium. Human Connectome Project Data Use Agreement. http://www.humanconnectomeproject.org/data/
} 
Sharing Terms and Conditions sets forth the expectations and procedures that investigators who want to submit their data to the NDA, which includes that all data submitted to the NDA should be de-identified and should be collected from subjects who consented to broadly share their data for research use. ${ }^{36}$ Users requesting access to NDA data are required to agree with terms and conditions laid out in its Data Use Certificate (DUC). ${ }^{37}$ The DUC allows access to data only for research purposes and prohibits reidentification of subjects or any further re-distribution of data. Users should also comply with regulatory and institutional requirements for protection of human subjects and safeguard data with security best practices to prevent unauthorized access to data. Moreover, they are required to provide an annual summary of research accomplishments achieved by using the NDA data and to report publications by creating an NDA Study that associates the publications and results with the underlying data in the NDA.

\section{Alzheimer's Disease Neuroimaging Initiative (ADNI)}

Alzheimer's Disease Neuroimaging Initiative (ADNI) is a longitudinal multicenter project that aims to develop clinical, imaging, genetic, and biochemical biomarkers for the early detection and tracking of Alzheimer's disease (Jack et al., 2008). For future sharing and secondary use of data, its data sharing policy requires an informed consent form used in each of the ADNI sites to include a statement that data collected from subjects will be de-identified and shared with ADNI members and the scientific community for research purposes. ${ }^{38}$ Investigators who want to gain access to data should first submit an application to the ADNI Data and Publication Committee (DPC). If applicants are qualified as members of the scientific community described in the consent form, the ADNI provides full, open access to all ADNI imaging and clinical data, which are limited data sets as defined under HIPAA, to applicants who agree to the conditions in the ADNI Data Use Agreement. ${ }^{39}$ After access to data is granted, applicants will receive annual requests to update the application information in addition to queries about manuscripts that resulted from research on shared data (e.g., title of manuscripts, information on authors of manuscripts, and status of each manuscript in development). Similar to the DUAs in other repositories, the ADNI DUA prohibits users from establishing the identity of subjects or contacting the subjects. No future disclosure of data beyond the uses outlined in the DUA and the application are allowed. Users should ensure to employ appropriate administrative, physical, and technical safeguards to prevent use or disclosure of the data other than as provided in the DUA. The DUA also requires acknowledging the ADNI as the data and funding source, but it further asks users to submit all manuscripts prior to submitting to a journal. The ADNI DPC will conduct an administrative review to confirm that the manuscript acknowledged the ADNI as outlined in the DUA. Finally, users should report any use or disclosure not provided for by the DUA, and non-compliance with the required updates will jeopardize future access to ADNI data.

\footnotetext{
36 The National Institute of Mental Health Data Archive (NDA). The NDA Data Sharing Terms and Conditions. https://s3.amazonaws.com/nda.nih.gov/Documents/NDA+Data+Sharing+Terms+and+Conditions+01.01.20.pdf

37 The National Institute of Mental Health Data Archive (NDA). The NDA Data Use Certificate. https://nda.nih.gov/ndapublicweb/Documents/NDA+Data+Access+Request+DUC+FINAL.pdf

${ }^{38}$ Alzheimer's Disease Neuroimaging Initiative (ADNI), ADNI Data Sharing and Publication Policy. http://adni.loni.usc.edu/wp-content/uploads/how_to_apply/ADNI_DSP_Policy.pdf

${ }^{39}$ Alzheimer's Disease Neuroimaging Initiative ( $\left.\overline{\mathrm{ADN}} \overline{\mathrm{DN}}\right), \mathrm{ADNI} \overline{\mathrm{Data}}$ Use Agreement. http://adni.loni.usc.edu/wp-content/uploads/how_to_apply/ADNI_Data_Use_Agreement.pdf.
} 


\section{iii. Alternative approaches}

Some research initiatives and databases have attempted to develop an alternative approach to sharing human subject data to better protect subjects' privacy or to respect subjects' autonomy on how their data should be used. For example, instead of sharing raw individual-level data, the Enhancing Neuroimaging Genetics through Meta-analysis (ENIGMA) provides analysis protocols to run at a local site and perform metaanalysis on results (e.g., summary statistics) returned from the local sites (Thompson et al., 2020; Thompson et al, 2014). The Collaborative Informatics and Neuroimaging Suite Toolkit for Anonymous Computation (COINSTAC) is another large-scale research consortium that offers tools for decentralized analysis which allows researchers to conduct both meta- and mega-analysis without pooling the data at one central place (Ming et al., 2017; Plis et al., 2016). Groups of users run common analyses on their local sites using their own data and the results of these analyses are then synchronized to the cloud and undergo aggregate analyses processes using all local data. The federated computing of COINSTAC also provides heightened privacy protection using advanced statistical algorithms, such as differential privacy, and this model may open up a way to gain access to data that researchers are unable to share due to local regulatory restrictions (Ming et al., 2017; White et al., 2020). However, it is important to note that federated computing could make data sharing more burdensome because it would require significant synchronized agreement efforts among all the sites to run the analysis locally or to change the protocol.

OpenHumans (openhumans.org) is a database that allows willing individuals or communities to share their own personal data for research purposes. Although most data currently shared in this database are genetic, demographic, or other personal data (e.g., social networking service data or data from digital healthcare apps), it may provide a useful reference for a potential new form of sharing neuroimaging data. In OpenHumans, individual contributors can opt in to certain activities, such as a research study, listed in the database. The recipients of data, investigators of the activities, are required to follow OpenHumans Activity Guidelines. ${ }^{40}$ Under the guidelines, the investigators should provide information on how data will be managed, such as whether data is de-identified or potentially identifiable, sensitivity of data, or other relevant privacy and security issues, to individuals to whom data pertains or to the IRB/ethics board. The investigators should also explain how they will handle data if an individual contributor decides not to participate in the activity any longer (e.g., deleting data). Some individuals make their data publicly available under the $\mathrm{CC} 0$ license, and investigators using these public data are subject to the requirements under OpenHumans Public Data Guidelines, which include preventing subject re-identification, acknowledging the data source, and providing a reference to these guidelines in any case of re-distributing the data. ${ }^{41}$

\section{Novel technological threats to data privacy in neuroimaging data sharing}

The potential risks of data sharing to privacy and confidentiality have long been acknowledged among researchers. Yet it has been believed that data sharing in research contexts is possible without compromising data privacy and confidentiality by adopting relatively simple data redaction methods and security measures. Removing specific information that can identify individual subjects from metadata has also been a common method of ensuring privacy protection. In neuroimaging studies, some researchers have blurred or removed individual subject's facial features from structural MR images (Bischoff-Grethe et al., 2007;

\footnotetext{
${ }^{40}$ OpenHumans, Activity guidelines, https://www.openhumans.org/community-guidelines/\#activity

${ }^{41}$ OpenHumans, Public Data Guidelines, https:/www.openhumans.org/community-guidelines/\#public-data
} 
Milchenko \& Marcus, 2013). Physically securing devices that store data (i.e., a computer or disks) and controlling access to data with password protection have also been perceived as reasonable practices of data security.

However, the emergence of advanced software tools and algorithms poses novel threats to data privacy because of their ability to re-identify subjects in neuroimaging datasets that have been thought to be de-identified. One of the well-known techniques reported to be able to invalidate conventional deidentification methods is facial reconstruction. In their 2019 study, Schwarz and colleagues showed that when only metadata are de-identified while facial features in structural images remain intact, it is possible to reconstruct individual subject's faces and re-identify them by matching the reconstructed faces with photos from social media or other resources using a facial recognition software (Schwarz et al., 2019). Other studies also have shown that even when neuroimaging data are defaced, it is still possible to reconstruct facial images. For example, Abramian and Eklund reported that generative adversarial networks (GANs), which is a popular tool for realistic image synthesis, can reconstruct facial features from defaced structural MR images (Abramian \& Eklund, 2019). The GANs-based algorithm developed in this study was able to convincingly restore face-blurred images, although it showed limited success for images in which facial features had been deleted. Re-identifying subjects with this technique could lead to a disclosure of related data or other information, such as other non-imaging data (e.g., cognitive scores), diagnosis or biomarkers of a disease, or participation in studies or clinical trials; however, the ability to reconstruct individual faces sufficiently well for reidentification has yet to be demonstrated, and it seems unlikely that the details of facial structure necessary for reidentification (e.g. eye spacing) could be reconstructed from skull structure after defacing.

Functional MRI fingerprinting, a technique that extracts individually unique patterns of functional images, can also be used to infer the identity of subjects. Studies have reported that identification using these brain signatures or fingerprints generates highly accurate results (Finn et al., 2017; Vanderwal et al., 2017; Ravindra, Drineas, \& Grama, 2021). Ravindra and Grama demonstrated that if one publicly available dataset is reidentified, then the individually unique functional signature can be matched across other datasets, which contain scans of the same subjects, leading to disclosure of the subjects' sensitive information in these datasets (Ravindra and Grama, 2019). Given the recent increase in sharing of multimodal datasets, as in HCP or ENIGMA that share genetic data along with neuroimaging data, MRI fingerprinting could potentially heighten the risks of revealing sensitive information about individual subjects. Combining multiple databases and collecting data from a longitudinal study could further exacerbate these risks to privacy. However, the accuracy of such methods decreases substantially when applied to larger datasets (Waller et al., 2017), suggesting that it may not be feasible to accurately reidentify individuals in real-world scenarios.

In addition, there have been significant advances in more generic ways to re-identify subjects based

on non-imaging data, using statistical modeling. For example, one study showed that by using a generative graphical model, individuals can be accurately re-identified with high likelihood even from heavily incomplete de-identified socio-demographic, survey, and health datasets (Rocher et al., 2019).

\section{Implications of novel threats to privacy in neuroimaging data sharing practices and policies}

Conventional de-identification methods, such as removal of direct identifiers in metadata or removing facial features in neuroimaging data, have been thought to be sufficient to protect subjects' privacy. In the United States, the regulatory protections for human subjects do not apply to de-identified neuroimaging data 
(Rothstein, 2010). Secondary analysis on de-identified neuroimaging data is not considered human subject research under the Common Rule, and thus, investigators are not required to comply with its requirements, informed consent and IRB review. De-identified data also do not constitute protected health information under the HIPAA. In other words, once data are de-identified, the current US regulatory system primarily relies on researchers to make ethical and judicious decisions on how and where to share their data (Ross et al., 2018). The basic assumption here is that researchers follow ethical principles and community norms in selecting data repositories and limiting subsequent use of data.

However, recent studies using novel tools and algorithms, such as facial reconstruction or MRI fingerprinting, have shown that any rigorously de-identified data sets still contain some risks of reidentification. To defend against this re-identification attack, researchers have been developing ways to counteract these tools and algorithms - for example, a new more effective defacing technique for structural images (Schwarz et al., 2021) or removal of signature patterns in functional images. Yet given the unprecedented pace of advancement in artificial intelligence and machine learning algorithms, it would be nearly impossible to ensure complete anonymization of data.

One might argue that there is a need for a more rigorous data protection regime to protect research subjects' privacy against these novel technological threats. Yet blanket regulation on data sharing might impose a greater burden on researchers who want to share low-risk data yet still provide insufficient protection for extremely sensitive data. Moreover, the fact that it is possible to re-identify subjects does not necessarily mean that it is likely to happen, especially given the current premature state of technology. The results from the recent studies might seem alarming, but these software tools and algorithms are still at an exploratory stage and have only been used for demonstration purposes. It should also be noted that reidentification per se can rarely cause harm to research subjects - that would require the actual misuse of data. Subjects would suffer harms only when sensitive information disclosed from re-identification is used to jeopardize the subjects' interests, as in the cases wherein a subject's disease state or biomarker derived from neuroimaging data is used to damage the subject's reputation or to put subjects' interests in peril in employment or health insurance contexts.

Unfounded fear or hype around what can be inferred from neuroimaging data could also dissuade not only potential subjects but also researchers from participating in neuroimaging research or sharing neuroimaging data. Choudhury and colleagues have argued that given the popular interest in neuroimaging that is "riddled with metaphors about 'mind-reading' capacities of neuroimaging and essentialistic hype about brain scans and personal identity," it is important to endorse critical understanding among the general public on the limits to what information about an individual can be inferred from neuroimaging data (Choudhury et al., 2014). The most salient risk to subjects that would occur through reidentification would be the use of those data to derive health-related information, such as the risk of developing a brain disorder such as Alzheimer's disease, or through the prediction of future behavior, such as criminal behavior. Thus, a fair assessment of the accuracy of brain imaging for such predictions is essential to realistically understanding the potential risks of reidentification. At present, there is reason to doubt that current neuroimaging methods will provide highly accurate prediction of future outcomes. First, there is evidence that reported predictive accuracy rates in the literature may be systematically inflated, due to the use of small samples along with analytic flexibility (Varoquaux, 2018; Poldrack, Huckins, \& Varoquaux, 2020). In addition, the real-world accuracy of prediction for rare outcomes (such as specific diseases or behavioral outcomes such as criminality) is heavily overestimated in studies that compare groups of equal sizes (Poldrack, Huckins, \& Varoquaux, 2020); measures that appear to be highly accurate in these settings are almost guaranteed to have very high rates of false positives when applied to the prediction of rare outcomes, 
or to have very low sensitivity if false positive rates are controlled. Thus, while it is impossible to predict the utility of future measures, the prediction of relatively rare outcomes is a fundamentally difficult problem; there is currently insufficient evidence to suggest that neuroimaging data can provide powerful predictions of future outcomes that could be used to discriminate against individuals.

Regardless of the level of risk, a more cautious approach needs to be developed to promote responsible sharing of neuroimaging data - for example, tiered control of data carefully calibrated to a realistic assessment of privacy risks (Ross et al., 2018). There has been a lack of regulatory oversight of data repositories, but the survey of data sharing practices in neuroimaging repositories showed that the repositories have already developed varying levels of access control and limitations on subsequent use of data, even de-identified, depending on the nature of data and the risks of harm associated with reidentification of data, among other relevant factors. Except in cases in which funding agencies require deposition of data in certain designated repositories, it can thus be said that the current ecosystem of neuroimaging data sharing offers a variety of options for researchers to share their data, including the alternative approaches of data sharing (e.g., CONISTAC or OpenHumans). As noted above, the decision on how to share data is left to the discretion of researchers, but developing guidelines such as the supplementary information to the NIH data sharing policy on desirable characters of data repositories would prove extremely valuable. These guidelines could also in turn inform neuroimaging repositories of the need to update their data sharing policy or data use agreement to better protect human subjects.

\section{Towards the ultimate protection against re-identification risks in neuroimaging data sharing}

Employing cutting-edge de-identification methods would substantially reduce the risk of reidentification. Controlling access to and subsequent use of neuroimaging data calibrated with the sensitivity of the data would further minimize the potential harm to subjects' privacy. Nevertheless, even with the best available privacy and security measures, it would be impossible to completely eliminate the risks of re-identification. If this remaining risk, however unlikely, were to materialize, it could potentially result in significant harm to subjects in some cases. Here we propose that potential harm of reidentification would be best addressed by introducing regulatory safeguards to prevent the misuse of information collected or disclosed as part of a research study.

As reviewed earlier, the enforcement mechanisms in the Common Rule and HIPAA do not provide proper legal ramifications for individuals who experience harm resulting from their participation in a research study, such as private cause of action regarding research-related harms. Given this lack of recourse on the side of research subjects, it is also not clear how to address potential harm to subjects caused by disclosure of research data in the context of data sharing under the current regulatory regime. In fact, the issue of using personal and sensitive information against individuals to whom the information pertains has received close attention from policy makers, and regulatory protections have been enacted to tackle this issue in other contexts. The most relevant example is the Genetic Information Non-discrimination Act (GINA). The GINA prohibits discrimination on the basis of genetic information in the context of health insurance and employment. ${ }^{42}$ Under GINA, it is unlawful for health insurers and employers to request,

\footnotetext{
42 The Patient Protection and Affordable Care Act (ACA) also contains provisions that prevent health insurers from discriminating against an individual (e.g., denying coverage or charging a higher premium) on the basis of an individual's participation in medical research. Yet here medical research is limited to a clinical trial conducted in relation to the prevention, detection, or treatment of a life-threatening disease or condition, such as a cancer. In
} 
require, or purchase genetic information with respect to an (potential) insured individual or employee. However, it is still possible that they might inadvertently obtain the genetic information of an insurance applicant or an employee. Given that it is not feasible to completely control the risk of disclosure, the protection under GINA is targeted at forbidding health insurers and employers from using one's genetic information for discriminative purposes rather than restraining them from having knowledge on the genetic information altogether. Adopting a regulatory scheme against malicious use of research data, similar to what GINA outlines, would offer ultimate protection for remaining risks of reidentification in the context of data sharing. Instead of imposing restrictions on the sharing of data, such a regulatory scheme - "The Neuroscience Information Non-Discrimination Act" - would enable us to chart a path to protect data privacy without undermining the substantial benefits of data sharing to human health. ${ }^{43}$

\section{Conclusion}

Neuroimaging is a scientific field that has greatly benefited from data sharing. Data sharing has been largely endorsed by researchers and neuroimaging studies on shared data have resulted in new scientific discoveries that deepen our understanding of the human brain and its functions. Yet for all its benefits, sharing neuroimaging data entails critical ethical and legal concerns, particularly regarding the privacy of subjects and confidentiality of data. In the United States, a multitude of federal and state regulations and policies provide protections for human subjects' identities and other sensitive information, including deidentification of data. However, recent advancements in software tools and algorithms to invalidate conventional de-identification methods pose heightened privacy risks in data sharing. Blanket regulations on data sharing could unduly hamper open science practice, so we should take a more cautious approach based on a realistic risk assessment, such as tiered control over data sharing based on privacy risks. In fact, existing neuroimaging databases have already developed varying levels of access control and limitations on the use of data depending on the sensitivity of the data. But given that it is currently under researchers' discretion to decide where to share data, it would be desirable for researchers to have some guidelines to adopt that promote more responsible sharing of neuroimaging data. Along with these guidelines, the remaining risks of re-identification could be addressed by introducing a regulatory safeguard against misuse of information collected or disclosed as part of a research study, similar to the safeguard in Genetic Information Non-Discrimination Act.

\section{Acknowledgement}

This research was supported by a grant from the National Institute of Mental Health of the National Institutes of Health under Award Number 3R24MH117179-03S. The content is solely the responsibility of the authors and does not necessarily represent the official views of the National Institutes of Health.

\footnotetext{
addition, the ACA does not prohibit "discrimination based on the disclosure of information gathered during research, but merely discrimination based on enrollment."

${ }^{43}$ Other scholars have proposed legislation akin to GINA regarding neuroscience data under different perspectives. For example, regarding the risks of using neuroscience data to compel suspects or defendants to produce selfincriminating evidence in criminal cases by decoding inner thoughts or memories, Farahany suggests adopting a GINA-like statutory approach for the protection of mental privacy, or cognitive liberty, against unwarranted government intrusions (Farahany, 2012).
} 


\section{References}

Abramian D. \& Eklund A. (2019). Refacing: reconstructing anonymized facial features using GANs. 2019 IEEE 16th International Symposium on Biomedical Imaging (ISBI 2019), 1104-1108, doi: https://doi.org/10.1101/447102

Alter, G., \& Gonzalez, R. (2018). Responsible practices for data sharing. Am Psychol, 73(2), 146-156. doi:10.1037/amp0000258

Bischoff-Grethe, A., Ozyurt, I. B., Busa, E., Quinn, B. T., Fennema-Notestine, C., Clark, C. P., .. . Fischl, B. (2007). A technique for the deidentification of structural brain MR images. Hum Brain Mapp, 28(9), 892-903. doi:10.1002/hbm.20312

Biswal, B. B., Mennes, M., Zuo, X. N., Gohel, S., Kelly, C., Smith, S. M., ...Milham, M. P. (2010). Toward discovery science of human brain function.Proceedings of the National Academy of Sciences of the United States of America, 107, 4734-4739.

Brakewood, B., \& Poldrack, R. A. (2013). The ethics of secondary data analysis: considering the application of Belmont principles to the sharing of neuroimaging data. Neuroimage, 82, 671-676. doi:10.1016/j.neuroimage.2013.02.040

Breeze, J. L., Poline, J. B., \& Kennedy, D. N. (2012). Data sharing and publishing in the field of neuroimaging. Gigascience, 1(1), 9. doi:10.1186/2047-217X-1-9

Choudhury, S., Fishman, J. R., McGowan, M. L., \& Juengst, E. T. (2014). Big data, open science and the brain: lessons learned from genomics. Front Hum Neurosci, 8, 239. doi:10.3389/fnhum.2014.00239

Cooper, J. A. \& McNair, L. (2015) Simplifying the complexity of confidentiality in research. J Empir Res Hum Res Ethics, 10, 100-102. doi: 10.1177/1556264614568783

Farahany, N. A. (2012). Incriminating thoughts, Stan L Rev 64, 351-408.

Finn, E. S., Scheinost, D., Finn, D. M., Shen, X., Papademetris, X., \& Constable, R. T. (2017). Can brain state be manipulated to emphasize individual differences in functional connectivity? Neuroimage, 160, 140-151. doi:10.1016/j.neuroimage.2017.03.064

Gorgolewski, K. J., Auer, T., Calhoun, V. D., Craddock, R. C., Das, S., Duff, E. P., . . Poldrack, R. A. (2016a). The brain imaging data structure, a format for organizing and describing outputs of neuroimaging experiments. Sci Data, 3, 160044. doi:10.1038/sdata.2016.44

Gorgolewski, K. J., Varoquaux, G., Rivera, G., Schwartz, Y., Sochat, V. V., Ghosh, S. S., . . Poldrack, R. A. (2016b). NeuroVault.org: A repository for sharing unthresholded statistical maps, parcellations, and atlases of the human brain. Neuroimage, 124( $\mathrm{Pt} \quad$ B), 1242-1244. doi:10.1016/j.neuroimage.2015.04.016

Health Information Portability and Accountability Act (1996). Pub. L. 104-191, § 110 Stat. 1936. Federal Policy for the Protection of Human Subjects, 45 C.F.R. $\S 46$.

Jack, C. R., Jr., Bernstein, M. A., Fox, N. C., Thompson, P., Alexander, G., Harvey, D., . . Weiner, M. W. (2008). The Alzheimer's Disease Neuroimaging Initiative (ADNI): MRI methods. $J$ Magn Reson Imaging, 27(4), 685-691. doi:10.1002/jmri.21049

Koch, V. G. (2015). A private right of action for informed consent in research. Seton Hall Law Rev, 45(1), 173-213. Retrieved from https://www.ncbi.nlm.nih.gov/pubmed/25911769

Mennes, M., Biswal, B. B., Castellanos, F. X., \& Milham, M. P. (2013). Making data sharing work: the FCP/INDI experience. Neuroimage, 82, 683-691. doi:10.1016/j.neuroimage.2012.10.064

Milchenko, M., \& Marcus, D. (2013). Obscuring surface anatomy in volumetric imaging data. Neuroinformatics, 11(1), 65-75. doi:10.1007/s12021-012-9160-3

Ming, J., Verner, E., Sarwate, A., Kelly, R., Reed, C., Kahleck, T., . . Calhoun, V. (2017). COINSTAC: Decentralizing the future of brain imaging analysis. F1000Res, 6, 1512. doi:10.12688/f1000research.12353.1 
National Commission for the Protection of Human Subjects of Biomedical and Behavioral Research. (1978) The Belmont report: ethical principles and guidelines for the protection of human subjects of research.

National Human Genome Research Institute. Genetic Information Nondiscrimination Act (GINA) of 2008. https://www.genome.gov/genetics-glossary/Genetic-Information-Nondiscrimination-Act

National Institute of Health (NIH) (2003). Final NIH statement on sharing research data. https:/grants.nih.gov/grants/guide/notice-files/NOT-OD-03-032.html

National Institute of Health (NIH) (2020a). Final NIH policy for data management and sharing. https:/grants.nih.gov/grants/guide/notice-files/NOT-OD-21-013.html

National Institute of Health (NIH) (2020b). Supplemental information to the NIH policy for data management and sharing: Selecting a repository for data resulting from NIH-sponsored research https:/grants.nih.gov/grants/guide/notice-files/NOT-OD-21-013.html

National Institute of Mental Health (NIMH) (2019). Notice of Data Sharing Policy for the National Institute of Mental Health. https://grants.nih.gov/grants/guide/notice-files/NOT-MH-19-033.html

Nichols, T. E., Das, S., Eickhoff, S. B., Evans, A. C., Glatard, T., Hanke, M., . . Yeo, B. T. (2017). Best practices in data analysis and sharing in neuroimaging using MRI. Nat Neurosci, 20(3), 299-303. doi:10.1038/nn.4500

Office of Human Research Protections (OHRP) (2008). Coded private information or specimens use in research, Guidance. https://www.hhs.gov/ohrp/regulations-and-policy/guidance/researchinvolving-coded-private-information/index.html

Peloquin, D., DiMaio, M., Bierer, B., \& Barnes, M. (2020). Disruptive and avoidable: GDPR challenges to secondary research uses of data. Eur J Hum Genet, 28(6), 697-705. doi:10.1038/s41431-0200596-X

Plis, S. M., Sarwate, A. D., Wood, D., Dieringer, C., Landis, D., Reed, C., . . Calhoun, V. D. (2016). COINSTAC: A Privacy Enabled Model and Prototype for Leveraging and Processing Decentralized Brain Imaging Data. Front Neurosci, 10, 365. doi:10.3389/fnins.2016.00365

Poldrack, R. A., \& Gorgolewski, K. J. (2017). OpenfMRI: Open sharing of task fMRI data. Neuroimage, 144(Pt B), 259-261. doi:10.1016/j.neuroimage.2015.05.073

Poldrack, R. A., Huckins, G., \& Varoquaux, G. (2020). Establishment of Best Practices for Evidence for Prediction: A Review. JAMA Psychiatry, 77(5), 534-540. doi:10.1001/jamapsychiatry.2019.3671

Poline, J. B., Breeze, J. L., Ghosh, S., Gorgolewski, K., Halchenko, Y. O., Hanke, M., . . Kennedy, D. N. (2012). Data sharing in neuroimaging research. Front Neuroinform, 6, 9. doi:10.3389/fninf.2012.00009

Presidential Commission for the Study of Bioethical Issues (2011). Moral science: Protecting participants in human subjects research, https://bioethicsarchive.georgetown.edu/pcsbi/node/558.html

Ravindra, V \& Grama, A. (2019). De-anonymization attacks on neuroimaging datasets, arXiv:1908.03260.

Ravindra, V., Drineas, P., \& Grama, A. (2021). Constructing Compact Signatures for Individual Fingerprinting of Brain Connectomes. Front Neurosci, 15, 549322. doi:10.3389/fnins.2021.549322

Ross, M. W., Iguchi, M. Y., \& Panicker, S. (2018). Ethical aspects of data sharing and research participant protections. Am Psychol, 73(2), 138-145. doi:10.1037/amp0000240

Rothstein, M. A. (2010). Is deidentification sufficient to protect health privacy in research? Am J Bioeth, 10(9), 3-11. doi:10.1080/15265161.2010.494215

Schwarz, C. G., Kremers, W. K., Therneau, T. M., Sharp, R. R., Gunter, J. L., Vemuri, P., . . Jack, C. R., Jr. (2019). Identification of Anonymous MRI Research Participants with Face-Recognition Software. N Engl J Med, 381(17), 1684-1686. doi:10.1056/NEJMc1908881

Schwarz, C. G., Kremers, W. K., Wiste, H. J., Gunter, J. L., Vemuri, P., Spychalla, A. J., . . Alzheimer's Disease Neuroimaging, I. (2021). Changing the face of neuroimaging research: Comparing a new MRI de-facing technique with popular alternatives. Neuroimage, 231, 117845. doi:10.1016/j.neuroimage.2021.117845 
The Brain Research Through Advancing Innovative Neurotechnologies (BRAIN) Initiative (2019).

Notice of data sharing policy for the BRAIN Initiative. https://grants.nih.gov/grants/guide/noticefiles/NOT-MH-19-010.html

The Presidential Commission for the Study of Bioethical Issues (2011) Moral science - Protecting participants in human subjects research https://bioethicsarchive.georgetown.edu/pcsbi/sites/default/files/Moral\%20Science\%20June\%202 012.pdf

Thompson, P. M., Jahanshad, N., Ching, C. R. K., Salminen, L. E., Thomopoulos, S. I., Bright, J., . . . Consortium, E. (2020). ENIGMA and global neuroscience: A decade of large-scale studies of the brain in health and disease across more than 40 countries. Transl Psychiatry, 10(1), 100. doi:10.1038/s41398-020-0705-1

Thompson, P. M., Stein, J. L., Medland, S. E., Hibar, D. P., Vasquez, A. A., Renteria, M. E., . . Alzheimer's Disease Neuroimaging Initiative, E. C. I. C. S. Y. S. G. (2014). The ENIGMA Consortium: largescale collaborative analyses of neuroimaging and genetic data. Brain Imaging Behav, 8(2), 153182. doi:10.1007/s11682-013-9269-5

U.S. Department of Health and Human Services (HHS) (2003a). Summary of the HIPAA PrivacyRule. http://www.hhs.gov/ocr/privacy/hipaa/understanding/summary/privacysummary.pd $\mathrm{f}$

U.S. Department of Health and Human Services (HHS) (2003b). Summary of the HIPAA Security Rule. http://www.hhs.gov/ocr/privacy/hipaa/understanding/srsummary.html

U.S. Department of Health and Human Services (HHS) (2008). Can a postsecondary institution be a "hybrid entity" under the HIPAA Privacy Rule? https://www.hhs.gov/hipaa/forprofessionals/faq/522/can-a-postsecondary-institution-be-a-hybrid-entity-under-hipaa/index.html

U.S. Department of Health and Human Services (HHS) (2013). Modifications to the HIPAA Privacy, Security, Enforcement, and Breach Notification Rules Under the Health Information Technology for Economic and Clinical Health Act and the Genetic Information Nondiscrimination Act; Other Modifications to the HIPAA Rules; Final Rule. https://www.govinfo.gov/content/pkg/FR-201301-25/pdf/2013-01073.pdf

U.S. Department of Health and Human Services (HHS) (2017a). Compliance \& Enforcement - How OCR enforces the HIPAA Privacy \& Security Rules. https://www.hhs.gov/hipaa/forprofessionals/compliance-enforcement/examples/how-ocr-enforces-the-hipaa-privacy-andsecurity-rules/index.html

U.S. Department of Health and Human Services (HHS) (2017b). Compliance \& Enforcement - State attorneys general. https://www.hhs.gov/hipaa/for-professionals/compliance-enforcement/stateattorneys-general/index.html?language $=$ en

Vanderwal, T., Eilbott, J., Finn, E. S., Craddock, R. C., Turnbull, A., \& Castellanos, F. X. (2017). Individual differences in functional connectivity during naturalistic viewing conditions. Neuroimage, 157, 521-530. doi:10.1016/j.neuroimage.2017.06.027

Van Essen, D. C., Smith, S. M., Barch, D. M., Behrens, T. E., Yacoub, E., Ugurbil, K., \& Consortium, W. U.-M. H. (2013). The WU-Minn Human Connectome Project: an overview. Neuroimage, 80, 6279. doi:10.1016/j.neuroimage.2013.05.041

Van Horn, J. D., \& Gazzaniga, M. S. (2013). Why share data? Lessons learned from the fMRIDC. Neuroimage, 82, 677-682. doi:10.1016/j.neuroimage.2012.11.010

Vanasse, T. J., Fox, P. M., Barron, D. S., Robertson, M., Eickhoff, S. B., Lancaster, J. L., \& Fox, P. T. (2018). BrainMap VBM: An environment for structural meta-analysis. Hum Brain Mapp, 39(8), 3308-3325. doi:10.1002/hbm.24078

Varoquaux, G. (2018). Cross-validation failure: Small sample sizes lead to large error bars. Neuroimage, 180(Pt A), 68-77. doi:10.1016/j.neuroimage.2017.06.061

Waller, L., Walter, H., Kruschwitz, J. D., Reuter, L., Muller, S., Erk, S., \& Veer, I. M. (2017). Evaluating the replicability, specificity, and generalizability of connectome fingerprints. Neuroimage, 158, 371-377. doi:10.1016/j.neuroimage.2017.07.016 
Westin, A. (1967). Privacy and Freedom (New York: Atheneum)

White, T., Blok, E., \& Calhoun, V. D. (2020). Data sharing and privacy issues in neuroimaging research: Opportunities, obstacles, challenges, and monsters under the bed. Hum Brain Mapp. doi:10.1002/hbm. 25120

World Medical Association. (2008) Declaration of Helsinki - Ethical principles for medical research involving human subjects. https://www.wma.net/policies-post/wma-declaration-of-helsinkiethical-principles-for-medical-research-involving-human-subjects/ 